\title{
A pandemia da COVID-19 trouxe desafios e novas possibilidades para a Fisioterapia no Brasil: estamos preparados?
}

\section{The COVID-19 pandemic brought challenges and new possibilities for Physiotherapy in Brazil: are we ready?}

${ }^{1}$ Autor para correspondência. Universidade do Estado de Santa Catarina (Florianópolis). Santa Catarina, Brasil. marlus.karsten@udesc.br ${ }^{2}$ Universidade do Estado de Santa Catarina (Florianópolis). Santa Catarina, Brasil. darlan.matte@udesc.br 3Universidade Católica de Pernambuco (Recife). Pernambuco, Brasil. flavio.andrade@unicap.br

A COVID-19 é uma síndrome clínica multissistêmica complexa que foi identificada em dezembro de 2019 na China após a detecção de uma série de casos de infecção respiratória grave com apresentações clínicas muito semelhantes a uma pneumonia viral ${ }^{1,2}$. A doença causada por um novo Coronavírus (SARS-CoV-2) se disseminou rapidamente para praticamente todas as partes do mundo e, em 11 de março, a Organização Mundial de Saúde a declarou como pandemia ${ }^{3}$. Após cinco meses, mais de 5,7 milhões de pessoas tiveram confirmação de infecção e mais de 356 mil pessoas morreram pela virose nos diversos continentes $^{4}$. No Brasil, o primeiro caso foi registrado em 25 de fevereiro. Desde então, o número de infectados confirmados ultrapassou os 411 mil e o número de casos fatais superou 25 mil vítimas, com elevada taxa de letalidade ${ }^{5}$.

Embora o acometimento seja de natureza multissistêmica, a COVID-19 afeta os indivíduos de forma diferente. Enquanto a grande maioria da população infectada (cerca de 80\%) é oligossintomática, uma parte tem sintomas clínicos importantes (15\%) e cerca de $5 \%$ dos infectados desenvolve a forma grave da doença, necessitando internação hospitalar para correção de hipoxemia grave ${ }^{6}$. As intervenções terapêuticas para correção desta disfunção são geralmente realizadas em unidades de terapia intensiva (UTI), com participação do fisioterapeuta intensivista e incluem, além da terapia medicamentosa, oxigenoterapia e suporte ventilatório (geralmente invasivo), podendo haver necessidade de posicionamento em prona, oxigenação por membrana extracorpórea (ECMO) e óxido nítrico inalatório ${ }^{7-12}$.

Dada a elevada complexidade dos casos graves de COVID-19, bem como a possibilidade de contágio nos ambientes hospitalares, os fisioterapeutas, assim como os demais profissionais envolvidos no processo assistencial hospitalar, têm sido expostos a grande carga de estresse físico e mental. Outros fatores que podem contribuir para essa sobrecarga são o inadequado dimensionamento das equipes de fisioterapia e a pouca disponibilidade de equipamentos de proteção individual (EPIs), de materiais e de dispositivos auxiliares para intervenção terapêutica que ocorrem em algumas instituições, assim como o grande volume de informações disseminadas, muitas vezes inconsistentes e sem respaldo científico adequado.

Nesse contexto, a Associação Brasileira de Fisioterapia Cardiorrespiratória e Fisioterapia em Terapia Intensiva - ASSOBRAFIR, considerando o seu papel de entidade representativa das especialidades da fisioterapia mais diretamente 
envolvidas no tratamento de pessoas com suspeita ou confirmação de COVID-19, criou um Comitê para assessoramento à Diretoria Executiva Nacional nas ações relativas à pandemia. Este Comitê tem monitorado as informações a respeito da COVID-19, especialmente aquelas relacionadas à atuação fisioterapêutica, organizado e divulgado os posicionamentos e informativos oficiais da ASSOBRAFIR, os quais podem ser consultados por toda a sociedade no site da associação (www.assobrafir.com.br/covid-19) ${ }^{13}$.

Por meio dessas publicações, desde o início da COVID-19 no Brasil, os fisioterapeutas respiratórios, cardiovasculares e intensivistas puderam acessar informações de qualidade, tanto por meio de comunicados oficiais e atividades on-line da própria ASSOBRAFIR como de outras associações e sociedades científicas, quanto pela divulgação de artigos de periódicos nacionais e internacionais. Além disso, os serviços de fisioterapia das principais instituições hospitalares têm desenvolvido protocolos ou rotinas de atendimento com base em evidências científicas, contribuindo para o sucesso do tratamento dos pacientes com COVID-19 nesses hospitais e naqueles que têm oportunidade de compartilhar informações. Assim, embora o tratamento de pacientes internados com COVID-19 ainda seja desafiador, o conhecimento dos principais recursos terapêuticos tem possibilitado a redução do estresse, mesmo que este permaneça elevado devido aos aspectos previamente elencados e à sobrecarga do sistema hospitalar em várias regiões do país ${ }^{13}$.

É importante ressaltar que, no contexto da pandemia, a atuação dos fisioterapeutas não se restringe apenas aos cuidados respiratórios dos pacientes internados com COVID-19, graves ou não, mas precisa também proporcionar intervenções com foco cardiovascular, metabólico e osteomioarticular, através de mobilização e exercícios terapêuticos precoces ou recursos como eletroestimulação neuromuscular e fotobiomodulação ${ }^{13}$. Além dos pacientes hospitalizados, os fisioterapeutas deveriam poder tratar os doentes com a forma leve da doença e certamente deverão tratar a grande quantidade de pacientes recuperados da fase aguda da COVID-19, que irão apresentar comprometimento funcional cardiovascular, respiratório e/ou metabólico crônico, além das alterações emocionais ${ }^{13-15}$.
Em relação aos pacientes com a forma leve (cerca de $80 \%$ do total), a orientação é para que mantenham isolamento domiciliar e sejam monitorados e atendidos pelo sistema de atenção primária à saúde, preferencialmente por teleconsulta e/ou telemonitoramento. Pacientes com comorbidades, como fragilidade ou doenças cardiovasculares, respiratórias, metabólicas ou neurológicas crônicas, possivelmente irão necessitar maior nível de acompanhamento, incluindo atendimento presencial. Nesses casos, os fisioterapeutas deverão usar EPIs adequados, orientar e seguir as recomendações para evitar contágio e infecção cruzada ${ }^{13}$.

Ainda no campo da assistência domiciliar, os fisioterapeutas poderão ter contato com pacientes que passaram pelo processo de desospitalização, mas que continuarão necessitando de suporte ventilatório invasivo ou não-invasivo. Nesses casos, deverão seguir as recomendações para abordagem fisioterapêutica hospitalar, especialmente em relação ao manejo da ventilação mecânica e à manutenção da função cardiorrespiratória e osteomioarticular. Os cuidados em relação à contaminação deverão ser mantidos, embora os pacientes desospitalizados provavelmente não devam mais estar na fase aguda da doença ${ }^{13}$.

Outro aspecto que necessita atenção dos fisioterapeutas é a necessidade de tratamento dos pacientes acometidos gravemente pela COVID-19 e que sobreviveram após um longo tempo de permanência na $\mathrm{UTI}^{14}$. O tempo prolongado de internação, a necessidade de ventilação mecânica invasiva e outros eventos deletérios inerentes à doença crítica certamente contribuirão para um curso clínico-funcional semeIhante ao de outras doenças respiratórias graves, e favorecerão o desenvolvimento da síndrome pós-terapia intensiva (PICS) ${ }^{16-19}$. A PICS se caracteriza por impacto significativo na função cardiopulmonar, na funcionalidade e mobilidade (incluindo perda de massa e função muscular, neuropatia, fraqueza muscular adquirida na UTI, e défice de equilíbrio), geralmente associado a comprometimento psicológico e cognitivo. Esta síndrome pode, inclusive, afetar os familiares dos pacientes, contribuindo para o desenvolvimento de ansiedade, depressão e transtorno de estresse pós-traumático, entre outros. Portanto, o sistema de saúde, o governo e os fisioterapeutas devem estar preparados para acolher, tratar e reabilitar os pacientes com PICS secundária à COVID-1918-19. 
Assim, os fisioterapeutas que trabalham em ambulatórios devem estar preparados para atenderem sobreviventes de COVID-19 grave com importante alteração da qualidade de vida relacionada à saúde e diversas alterações funcionais, como: sintomas respiratórios, capacidade de exercício reduzida, fraqueza e fadiga muscular periférica e respiratória, alterações posturais e de equilibrio, lesões de pele, entre outras. Caberá a estes profissionais participar ativamente do processo de reabilitação pós-COVID-19, que sabidamente contribui para melhorar a capacidade física e funcional, a autoeficácia e a prontidão para o exercício ${ }^{17}$.

Um dos aspectos positivos associados aos períodos de crise sanitária, como a que vivemos, é a aceleração do desenvolvimento de recursos terapêuticos. Nesse sentido, vale destacar o importante papel das modalidades de consulta, monitoramento e atendimento remotos, os quais foram recentemente regulamentados $^{20}$ e passaram a ser explorados como alternativa. É possível que o aprimoramento destes recursos passe a integrar as possibilidades de atuação num futuro próximo, pois as evidências relacionadas ao que se convencionou chamar de telerreabilitação estão em expansão. Ainda no campo da pesquisa e inovação tecnológica, vale destacar o papel de fisioterapeutas em equipes multiprofissionais que estão trabalhando para desenvolver equipamentos de ventilação mecânica em território nacional ${ }^{21,22}$.

Com isso, são evidentes a necessidade e a importância dos fisioterapeutas na linha de frente e na retaguarda do enfrentamento à pandemia da COVID-19. Possivelmente a fisioterapia brasileira ainda não havia experimentado um período com tamanhos desafios e possibilidades de projeção e valorização, como este que estamos vivendo. Atuamos nos diversos cenários da pandemia (atenção básica, atuação hospitalar, desospitalização, atenção domiciliar, ambulatorial e remota), além de participar ativamente de atividades de pesquisa, inovação, ensino e desenvolvimento de políticas públicas. Vale destacar a participação ativa e diferenciada dos fisioterapeutas intensivistas junto aos pacientes graves, reforçando a importância da permanência desses profissionais de forma ininterrupta nas UTIs, perfazendo as 24 horas diárias. Cabe, ainda, aos fisioterapeutas documentarem sua atuação e publicarem os resultados de seus procedimentos e intervenções. Muito provavelmente o país e o mundo estarão diferentes após a pandemia, e os desafios impostos aos fisioterapeutas poderão se converter em estímulos para o aprimoramento e desenvolvimento profissional e das relações profissionais. Sim, estamos preparados para isso e o faremos com responsabilidade e compromisso social e ético.

\section{Referências}

1. Yu P, Zhu J, Zhang Z, Han Y. A familial cluster of pneumonia associated with the 2019 novel coronavirus indicating personto-person transmission: a study of a family cluster. J Infect Dis. 2020;221(11):1757-1761. doi: 10.1093/infdis/jiaa077

2. Huang C, Wang Y, Li X, Ren L, Zhao J, Hu Y et al. Clinical features of patients infected with 2019 novel coronavirus in Wuhan, China. Lancet. 2020;395:497-506. doi: 10.1016/S0140-6736(20)30183-5

3. World Health Organization. Timeline - COVID-19. [Internet]. 2020. Available from: https://www.who.int/news-room/detail/2704-2020-who-timeline---covid-19

4. COVID-19 Dashboard by the Center for Systems Science and Engineering (CSSE) at Johns Hopkins University (JHU). [Internet]. Available from: https://gisanddata. maps.arcgis.com/apps/opsdashboard/index.html\#/ bda7594740fd40299423467b48e9ecf6

5. Ministério da Saúde (BR). COVID19 Painel Coronavírus. [Internet]. Available from: https://covid.saude.gov.br/

6. Zhou F, Yu T, Du R, Fan G, Liu Y, Liu Z et al. Articles Clinical course and risk factors for mortality of adult inpatients with COVID-19 in Wuhan, China: a retrospective cohort study. Lancet. 2020;395:1054-62. doi: 10.1016/S0140-6736(20)30566-3

7. World Health Organization. Clinical management of severe acute respiratory infection (SARI) when COVID-19 is suspected. [Internet]. 2020. Available from: https://www.who.int/publicationsdetail/clinical-management-of-severe-acute-respiratory-infectionwhen-novel-coronavirus-(ncov)-infection-is-suspected

8. Wang L, Li X, Yang Z, Tang X, Yuan Q, Deng L et al. Semirecumbent position versus supine position for the prevention of ventilator-associated pneumonia in adults requiring mechanical ventilation. Cochrane Database Syst Rev. 2016;(1):CD009946. doi: 10.1002/14651858.CD009946.pub2

9. Chacko B, Peter JV, Tharyan P, John G, Jeyaseelan L. Pressurecontrolled versus volume-controlled ventilation for acute respiratory failure due to acute lung injury (ALI) or acute respiratory distress syndrome (ARDS). Cochrane Database Syst Rev. 2015;(1):CD008807. doi: 10.1002/14651858.CD008807.pub2

10. Amato MBP, Meade MO, Slutsky AS, Brochard L, Costa ELV, Schoenfeld DA et al. Driving pressure and survival in the acute respiratory distress syndrome. N Engl J Med. 2015;372:747-55. doi: $10.1056 / \mathrm{NEJMSa1410639}$ 
11. Santa Cruz R, Rojas Jl, Nervi R, Heredia R, Ciapponi A. High versus low positive end-expiratory pressure (PEEP) levels for mechanically ventilated adult patients with acute lung injury and acute respiratory distress syndrome. Cochrane Database of Systematic Reviews. 2013;(6):CD009098. doi: 10.1002/14651858. CD009098.pub2

12. Guérin C, Reignier J, Richard J, Beuret P, Gacouin A, Boulain $T$ et al. Prone Positioning in Severe Acute Respiratory Distress Syndrom. N Engl J Med. 2013;368(23):2159-2168. doi: 10.1056/ NEJMoa1214103

13. Associação Brasileira de Fisioterapia Cardiorrespiratória e Fisioterapia em Terapia Intensiva. Comunicação Oficial ASSOBRAFIR COVID-19 [Internet]. Available from: www.assobrafir. com.br/covid-19

14. Spruit MA, Holland AE, Singh SJ, Troosters T. Report of an adhoc international task force to develop an expert-based opinion on early and short-term rehabilitative interventions (after the acute hospital setting) in COVID-19 survivors (Version April 3, 2020). [Internet]. 2020. Available from: https://www.ersnet.org/ covid-19-blog/covid-19-and-rehabilitation

15. Ceravollo MG, Sire A, Andrenelli E, Negrini F, Negrini S. Systematic rapid living review on rehabilitation needs due to COVID-19: update to March 31st 2020. Eur J Phys Rehabil Med. 2020. doi: $10.23736 /$ S1973-9087.20.06329-7

16. Edwards E. Post intensive-care syndrome: Why some COVID-19 patients may face problems even after recovery. People who remain in the ICU for weeks may end up with memory problems and trouble thinking clearly. [Internet]. 2020. [cited $2020 \mathrm{Apr}$ 9]. Available from: http://city-countyobserver.com/postintensive-care-syndrome-why-some-covid-19-patients-may-faceproblems-even-after-recovery/

17. Koh GC, Hoenig H. How should the rehabilitation community prepare for 2019-nCoV? Arch Phys Med Rehabil 2020;101(6):10681071. doi: 10.1016/j.apmr.2020.03.003

18. Colbenson GA, Johnson A, Wilson ME. Post-intensive care syndrome: impact, prevention, and management. Breathe (Sheff). 2019;15(2):98-101. doi: 10.1183/20734735.0013-2019

19. Stam HJ, Stucki G, Bickenbach J. COVID-19 and post intensive care syndrome: a call for action. J Rehabil Med 2020;52(4):jrm00044. doi: 10.2340/16501977-2677

20. Brasil. Resolução COFFITO n 516, de 20 de março de 2020. Dispõe sobre a suspensão temporária do Artigo 15, inciso II e Artigo 39 da Resolução COFFITO no 424/2013 e Artigo 15, inciso II e Artigo 39 da Resolução COFFITO no 425/2013 e estabelece outras providências durante o enfrentamento da crise provocada pela Pandemia do COVID-19. Diário Oficial da União $n^{\circ} 56$. Seção 1, de 23/03/2020. Brasília/DF.
21. Instituto Federal de São Paulo. Pesquisadores do IFSP e IFCE estão desenvolvendo protótipo de respirador. [Internet]. 2020. Available from: https://www.ifsp.edu.br/noticias/1456pesquisadores-do-ifsp-e-ifce-estao-desenvolvendo-prototipo-derespirador

22. Conselho Regional de Fisioterapia e Terapia Ocupacional. Fisioterapeutas participam do desenvolvimento de respirador de baixo custo em Bento Gonçalves. [Internet]. 2020. Available from: http://www.crefito5.org.br/noticia/fisioterapeutas-participamdo-desenvolvimento-de-respirador-de-baixo-custo-em-bentogoncalves 